Autonomous monitoring of control hardware to predict off-normal conditions using NIF automatic Alignment Systems

A. Awwal, K. Wilhelmsen, R. Leach, V. Miller Kamm, S. Burkhart, R. Lowe-Webb, S. Cohen

July 28, 2011

IAEA 8th Technical Meeting

San Francisco, CA, United States

June 20, 2011 through June 24, 2011 
This document was prepared as an account of work sponsored by an agency of the United States government. Neither the United States government nor Lawrence Livermore National Security, LLC, nor any of their employees makes any warranty, expressed or implied, or assumes any legal liability or responsibility for the accuracy, completeness, or usefulness of any information, apparatus, product, or process disclosed, or represents that its use would not infringe privately owned rights. Reference herein to any specific commercial product, process, or service by trade name, trademark, manufacturer, or otherwise does not necessarily constitute or imply its endorsement, recommendation, or favoring by the United States government or Lawrence Livermore National Security, LLC. The views and opinions of authors expressed herein do not necessarily state or reflect those of the United States government or Lawrence Livermore National Security, LLC, and shall not be used for advertising or product endorsement purposes. 


\title{
Autonomous monitoring of control hardware to predict off-normal conditions using NIF automatic Alignment Systems
}

\author{
Abdul A. S. Awwal, Karl Wilhelmsen, Richard Leach, Vicki Miller Kamm, Scott Burkhart, Roger \\ Lowe-Webb, and Simon Cohen
}

Lawrence Livermore National Laboratory, Livermore, CA 94550

\begin{abstract}
The National Ignition Facility (NIF) is a high power laser system capable of supporting high-energy-density experimentation as a user facility for the next 30 years. In order to maximize the facility availability, preventive maintenance enhancements are being introduced into the system. An example of such an enhancement is a camerabased health monitoring system, integrated into the automated alignment system, which provides an opportunity to monitor trends in measurements such as average beam intensity, size of the beam, and pixel saturation. The monitoring system will generate alerts based on observed trends in measurements to allow scheduled pro-active maintenance before routine off-normal detection stops system operations requiring unscheduled intervention.
\end{abstract}

Keywords: Automated laser alignment, off-normal images, uncertainty, trend monitoring, preventive maintenance.

\section{Introduction}

The National Ignition Facility (NIF) is a 192-beam laser system including thousands of control points such as electro-mechanical devices and sensor cameras that are used to aim and focus the beams along the beam path onto the experimental target. An automatic alignment (AA) system was developed to process CCD sensor images of the laser beams taken at various locations. The control system uses results of the processing to adjust motorized mirrors within a series of control loops until pre-defined alignment criteria are satisfied [1-3]. In addition to providing alignment information, these cameras offer an eye into many aspects of the system health.

Parameters are routinely measured such as average intensity of the beam, size of the illuminated region, minimum and maximum image values and count of saturated pixels, etc. The data also contains useful information about the health of the NIF laser system that can be presented to operations personnel. For example, automated trend analysis of motor adjustments could be used to trigger a notification to system operators that they should investigate the cause of any deteriorating conditions (e.g., excessive drift could possibly be attributed to a failing mechanical device). Monitored conditions are compared against nominal values such that when the deviations exceed certain limits, an offnormal alert is raised. The automated health monitoring system will avoid the difficulty of data mining for trends within a large image history. Additional evaluation of sensor and control data during normal operations to detect off-normal conditions or trends is a technique that can be applied to other large-scale systems. In this paper, we describe the NIF health monitoring system, the automated algorithms and the integrated control system used to increase the overall availability of NIF's optics inspection system, which is undergoing testing and initial deployment. We will also discuss extending the system to monitor health in other areas of the NIF laser.

\section{Motivation}

The original goal of automated alignment was to align the laser beams, using available alignment images regardless of quality. The challenge was to make the image processing robust enough to deal with any imaging situation. Later, it was found that some of the worst alignment images were indicative of optomechanical failure. The goal was then to detect these cases, called off-normal images, and stop the alignment until the operator manually resolved the situation. However, during commissioning of new alignment loops or new hardware, off-normal images were expected. A commissioning mode was created to temporarily disable the off-normal checks. Routinely, the off-normal parameters are applied uniformly to all beams. However, certain beams may have slightly different normal condition. Individual beams were tailored to offer special processing with slightly different criterion.

Recently, a flexible off-normal detection enhancement involving a database of nominal values for key parameters of different beams was implemented. Off-normal detection compares each beam measurement against the respective value from the set of nominal values and produces an alert if the deviation exceeds a defined percentage of the nominal value. Additionally, the current values of all the beams are saved in a separate file with appropriate time stamp for later inspection.

In this paper, we propose to extend the beamdependent off-normal detection to include trending where current measurement is not only compared to the nominal values but also to the previous measurement of the same type in order to detect degrading trend of parameters developing in the laser system. This detection is expected to maximize system availability by initiating pro-active maintenance on the deteriorating hardware.

This work was performed under the auspices of the U.S. Department of Energy by Lawrence Livermore National Laboratory under Contract DE-AC52-07NA27344

Author's email: awwal1 @llnl.gov 


\section{Progress in Off-normal detection}

The closed loop automated alignment system concurrently performs alignment of 192 beams to the target chamber center. The normal operation of the automated alignment system is performed as follows: alignment images are acquired from a charge-coupled device (CCD) imaging camera producing both reference and measurement images. The reference image is used to provide the desired fiducial that is used by the alignment system. The alignment of the measured image with the reference is accomplished by using the dedicated control loops that adjust pointing mirror stepping motors until the deviations between both reference and measured positions are within acceptable limits [4]. The close loop control is interrupted when an anomalous or off-normal event occurs and is notified to operations personnel by the AA algorithm.

The heart of the alignment system is the automatic alignment algorithms [5] which process both the reference and beam images to find the position. All image processing algorithms undergo a three step process in order to provide reliable position information from the video image as depicted in Figure 1. The offnormal processor detection ensures that the image that is captured is a valid image whose position data can be relied upon to make adjustments [6]. The algorithm generates the position data and an uncertainty detector generates a number indicating the reliability of the position measurement as it relates to the image quality. An uncertainty over a certain limit transfers the automatic alignment to a manual operator assisted mode.

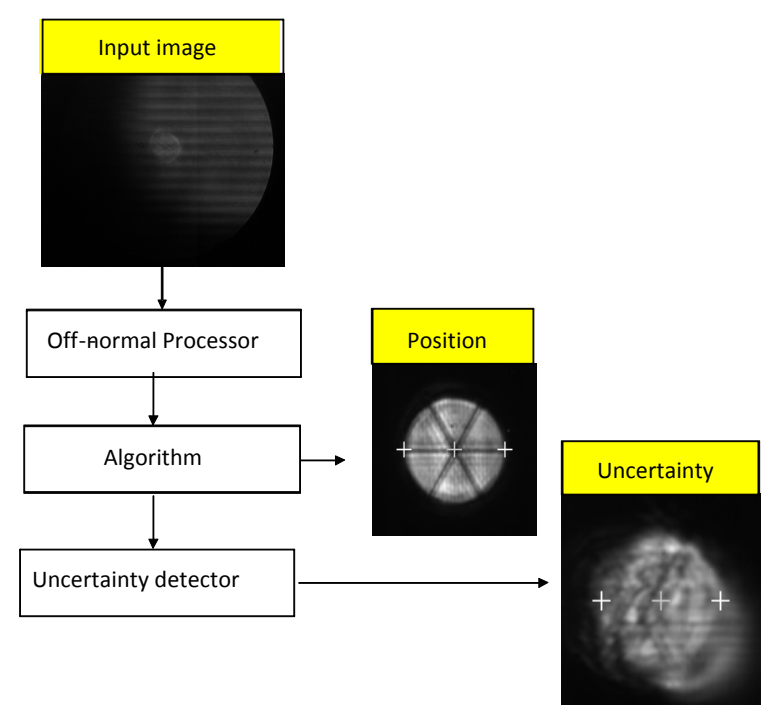

Fig.1 Image processing algorithm process flow

Examples of normal and off-normal images are shown in Figure 2. An "off-normal" image may occur due to component malfunctions (sticky mirror, stuck shutter, stepping motor failure, component failure, wavefront distortion etc.). When the off-normal detector detects an off-normal image, a high uncertainty is produced by the algorithm, which shuts down the automatic alignment until the control room operators intervene to examine and resolve the situation. This process greatly minimizes the risk of accidental misalignment caused by component malfunction.
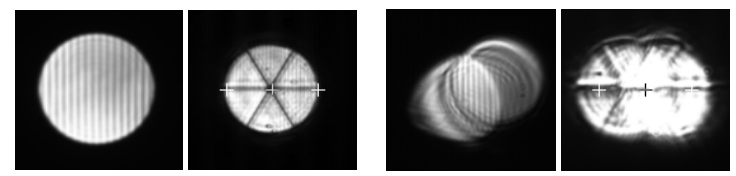

Fig.2 Example of normal and off-normal beam images.

The most simple off-normal check is detecting whether the image has any information content at all. Examples include checking whether an image is all black or all white, which could happen due to a camera malfunction. When these two cases are encountered the algorithm stops further processing of the image and produces a high-uncertainty value which forces the AA control to stop aligning. For other image/beam characteristics, a more graded approach of uncertainty detection is implemented.

Another important beam characteristic for the image types shown in Figure 2 is the width of the beam. The width may be measured around various angular positions through the center of the beam. The criterion is based on a tolerance threshold, $\mathrm{T}$ is as shown in Eq. 1:

$\left|L(\theta)-\mu_{L}\right| \leq \mathrm{T}$

for $L(\theta)$ the width (in pixels) of a line at angle, $\theta$, which could be $0^{\circ}, 45^{\circ}, 90^{\circ}$ or $135^{\circ}$ and $\mu_{L}$ is the expected line width. A typical value of $\mathrm{T}$ could be 20 , where the value of width could be 70 pixels radius. Based on the above check, the two right images of Figure 2 can be rejected. Examples of other distance criteria applied to various image are distance between fiducials or edges as depicted in Figure 3. These

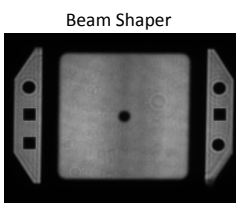

ISP_LM3 beam
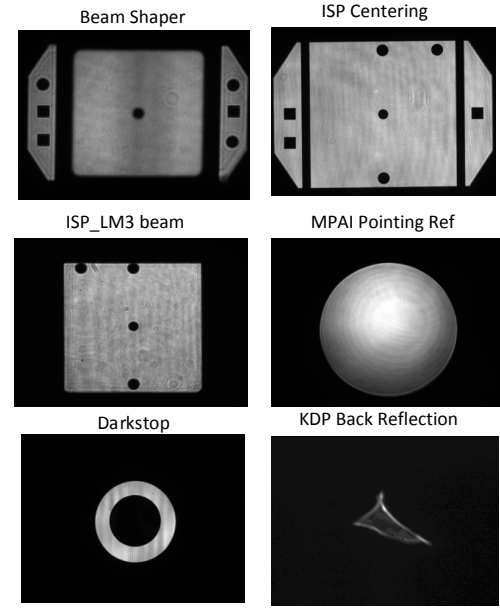

MPAl Pointing Ref

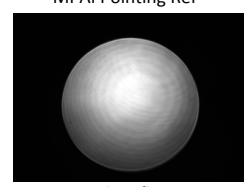

KDP Back Reflection
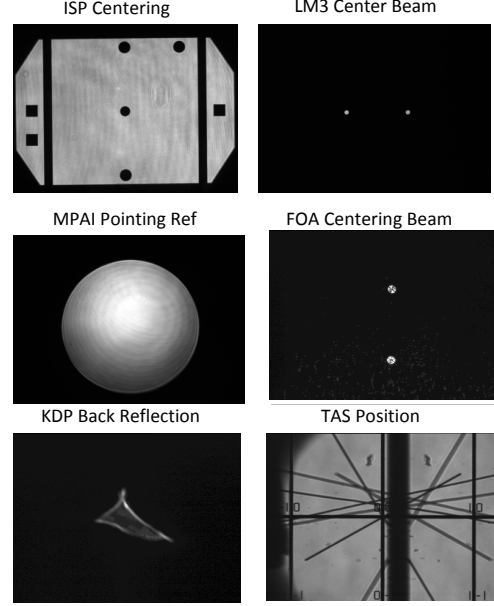

FOA Centering Beam

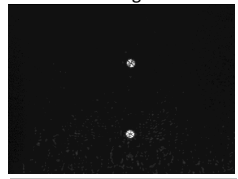

TAS Position

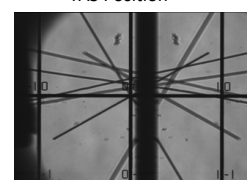

Fig.3 Examples of beam images requiring off-normal check

criteria may catch conditions where the beam is truncated or there is a false fiducial present. For BeamShaper types of images, the horizontal and vertical distances between the square and circular fiducials on the wings are measured. For LM3 centering beams, the 
distance between the two spots are measured to ensure that the correct light sources are used for alignment. For pinhole type of beams (as shown in Fig. 2) the size is measured to ensure the beam is not cutoff by an aperture.

However, if these images are acquired during the commissioning of new hardware, the system allows offnormal to be over-ridden in a mode known as commissioning mode. In this mode, the AA system keeps operating with the off-normal images which are expected during commissioning.

Other important off-normal parameters are nominal, maximum and minimum image intensities which can detect a beam that is gradually deteriorating. There are multiple reasons why the nominal average beam intensity is important. If the beam intensity is low, then the position data may be less reliable or accurate and when the intensity is too low it may also cause algorithm failure. Low intensity, below a nominal value, indicates that the camera integration time may be low or camera attenuation too high or the source life is nearing its end. Generally, the nominal intensity should be above a certain minimum below which the magnitude of the measurement uncertainty is more than the alignment tolerance for the specific loop. As shown in Eq. (2), this measured value could be the maximum or the average intensity of the illuminated area.

$I_{\text {measured }}>I_{\text {min }}$

When the above condition is violated, an off-normal condition may be flagged.

\section{SIDE optics inspection}

The Side Illuminated Damage Evaluation (SIDE) system, illuminates a Target Chamber Vacuum Window (TCVW) from two adjacent sides and acquires an image of the scattered light to determine if there is damage on the optic. In this acquisition there are integrity checks to ensure that the light level of the two illumination strips are appropriate. An image of a TCVW optic is shown in Figure 4 (different TCVW optics are rotated clockwise or counter-clockwise slightly by the imaging system). There are 96 of these SIDE Systems on NIF. On each TCVW two vertical light regions (called strip 1) are generated from one horizontal strip of light bulbs. Strip 2 is generated from a second vertical source of light bulbs as shown in Figure 5. The current requirement dictates that there should be at least 1500 pixels with a gray level above 250 count. When the number of bright pixels above the threshold 250 falls below 1500, it is declared off-normal. When an off-normal failure happens, the illumination strips are removed and replaced. It was found that the light sources for different beam lines vary in intensity; therefore practically it would be better to have different criteria for different beam lines. In order to set the base line, the nominal intensity of all the images is measured and saved in a Table as shown in left side of Figure 6.
Figure 7 shows a block diagram representation of the whole process. During real time data acquisition, the offnormal tests compare the measured values against the nominal values and record the deviation from the nominal value. When the deviations exceed a predefined limit, the tests fail. All pass/fail performances based on these comparisons are recorded.

Notice that the time-stamped measurements provide an avenue for further extension as described in the next section. As a failed component is replaced, the nominal values file is updated to record the new nominal value.

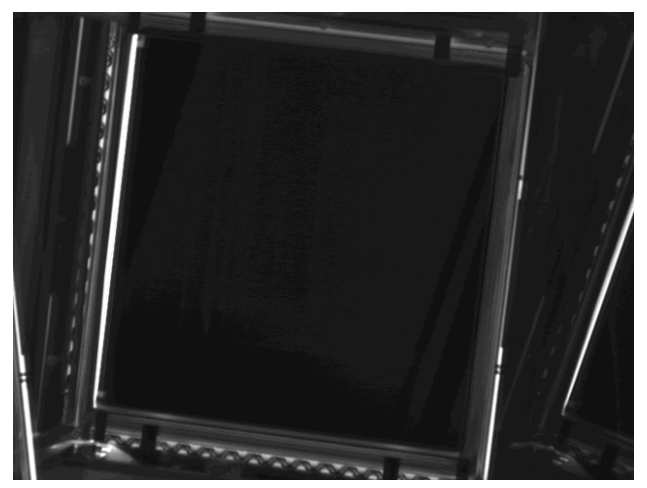

Fig.4 SIDE High image

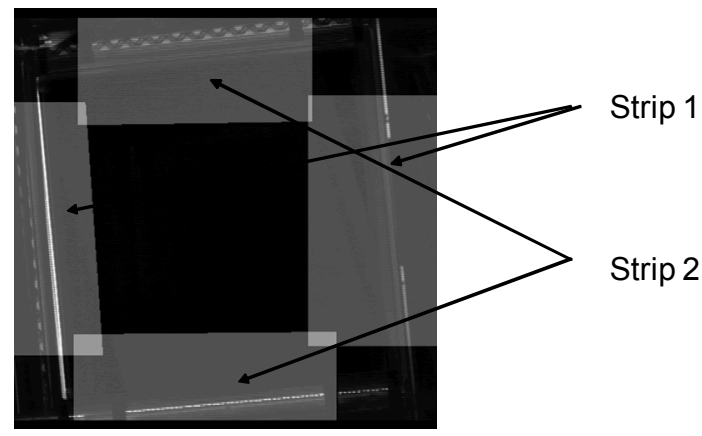

Fig.5 SIDE High image showing strip 1 and strip 2 locations.

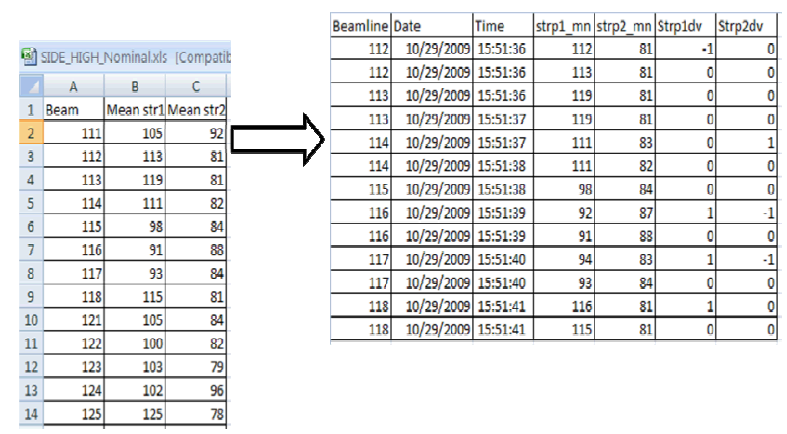

Fig. 6 The table on the left is the file containing the nominal mean intensities of strip 1 and strip 2 for various beam lines for the SIDE High image. The right image shows measured values at different times with deviations from the nominal values. 


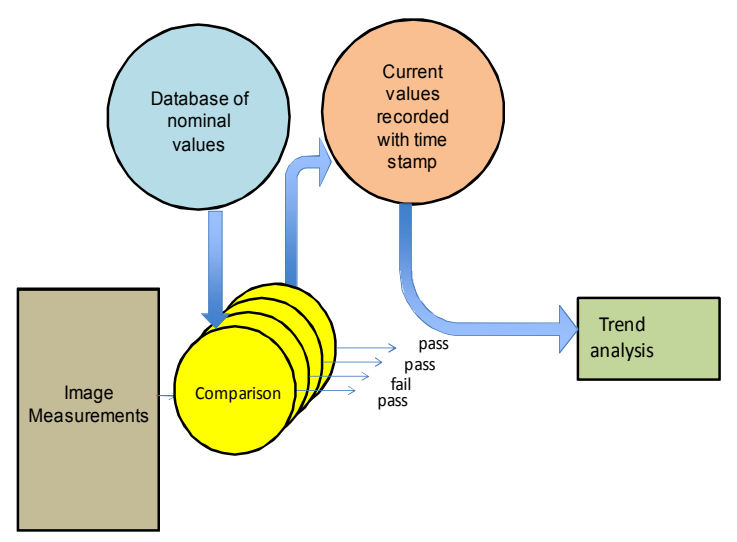

Fig.7 Proposed system showing trend analysis

\section{Predictive Monitoring}

In order to take advantage of the enormous amount of data generated during NIF alignment, we propose to extend the off-normal detection system implemented as shown in Figure 7 to include trend analysis. Adding this capability will allow us better system monitoring and permit planned maintenance on the system. Consequently, this will increase availability of the NIF laser while reducing downturn by avoiding failure before it happens.

The trend monitoring can be explained as follows. Imagine one of the beam characteristics being measured as shown in Figure 8. Also we assume the nominal value of this quantity (beam intensity) is around 200 (for an 8-bit image). When this quantity falls below a value of 100, it is declared off-normal, which happens approximately after 80 units of time. By measuring the trend, it may be possible to detect the upcoming offnormal event only after 20 units of time. The magnitude of the slope may alert us as to how much time is left before the off-normal event will occur. This gives us sufficient time to schedule maintenance well before the $80^{\text {th }}$ mark. Component selection may be done more prudently if this trend is noticed for devices from a certain supplier.

\section{Trend in measurements}

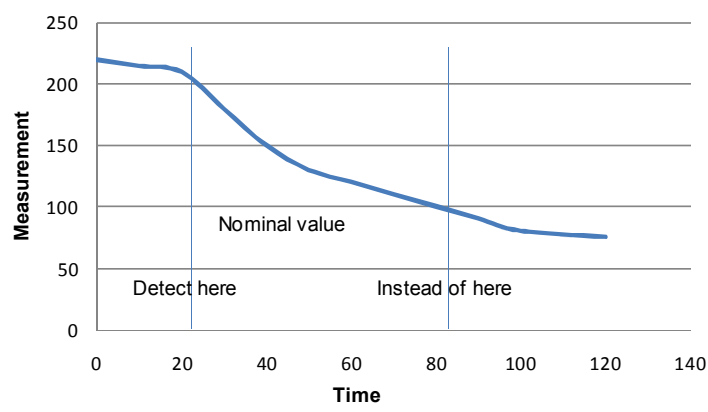

Fig.8 Trend data showing possible deterioration.
The trend threshold will have to be determined and also the nature of alert. The current off-normal alerts are immediate and stop the alignment when it happens. The trend alerts may go to the control room personnel in a different manner, where all the decisions are made regarding servicing a particular physical hardware.

\section{Conclusion}

As NIF moves into the mode of a user facility, we need to employ all system measurements to track the health of the laser system. The concept can be extended to new major nuclear power plants, mining and many other similar high risk, high payoff, high consequence situations where camera (or any other sensor) based system can be used to perform predictive monitoring.

\section{Acknowledgments}

We appreciate the editing advice and guidance from Steve Azevedo, Gordon Brunton, Peg Folta, Larry Lagin and Chris Marshall.

\section{References}

[1] R. Zacharias, N. Beer, E. S. Bliss, S. Burkhart, S. Cohen, S. Button, R. Van Atta, S. Winters, J. Salmon, M. Latta, C. Stoiz, D. Pigg and T. Arnold, "Alignment and wavefront control systems of the National Ignition Facility," Opt. Eng., 43 (12), pp. 2873-2884, (2004).

[2] S. C. Burkhart, E. Bliss, P. Di Nicola, D. Kalantar, R. Lowe-Webb, T. McCarville, D. Nelson, T. Salmon, T. Schindler, J. Villanueva, and K. Wilhelmsen, "National Ignition Facility system alignment," Appl. Opt. 50, 11361157 (2011)

[3] K. Wilhelmsen, A. Awwal, W. Ferguson, B Horowitz, V. Miller Kamm, C. Reynolds, "Automatic Alignment System For The National Ignition Facility", pp. 486-490, Proceedings of 2007 International Conference on Accelerator and Large Experimental Control Systems (ICALEPCS07), October 15-19, Knoxville, Tennessee

[4] J. V. Candy, W. A. McClay, A. A. S. Awwal, and S. W. Ferguson, "Optimal position estimation for the automatic alignment of a high-energy laser," Journal of Optical Society of America A, 22, pp. 1348-1356, 2005.

[5] A. A. S. Awwal, Wilbert A. McClay, Walter S. Ferguson, James V. Candy, Thad Salmon, and Paul Wegner, "Detection and Tracking of the Back-Reflection of KDP Images in the presence or absence of a Phase mask," Applied Optics, 45, 3038-3048, 2006.

[6] J. V. Candy, A. A. S. Awwal, W. McClay, S. C. Burkhart, "Detection of off-normal images for NIF automatic alignment," in Photonic Devices and Algorithms for Computing VII, edited by K. Iftekharuddin and A. A. S. Awwal,, Proc. of SPIE Vol. 5907, pp. 59070B-1 59070B-12, 2005. 